\title{
Laying the foundations of programmed cell death
}

\author{
A Letai ${ }^{\star *, 1,2}$ and L Scorrano ${ }^{\star, 3}$ \\ ${ }^{1}$ Department of Medical Oncology, Dana-Farber Cancer Institute, Boston, MA, \\ USA \\ 2 Department of Medicine, Harvard Medical School, 44 Binney Street, Boston, \\ MA 02115, USA \\ ${ }^{3}$ Venetian Institute of Molecular Medicine, Dulbecco-Telethon Institute, Via \\ Orus 2, 35129 Padova, Italy \\ * Corresponding authors: A Letai, Department of Medical Oncology, Dana- \\ Farber Cancer Institute, Department of Medicine, Harvard Medical School, 44 \\ Binney Street, Boston, MA 02115, USA. \\ E-mail: anthony_letai@dfci.harvard.edu and L Scorrano, Venetian Institute of \\ Molecular Medicine, Dulbecco-Telethon Institute, Via Orus 2, 35129 Padova, \\ Italy. Tel: + 39049792 3221; Fax: + 39049792 3271; \\ E-mail: luca.scorrano@unipd.it
}

\section{Cell Death and Differentiation (2006) 13, 1245-1247. doi:10.1038/sj.cdd.4401999}

A year has passed since the death of Stan Korsmeyer (Figure 1). His energetic, productive and generous life, and his many contributions to the lives and careers of others, has been suitably eulogized in many prominent settings. It seems appropriate that an issue in a journal devoted to the study of programmed cell death (PCD) should put in context a summary of the seminal contributions Stan made during his work as a founder of the field of cell death research. A review of his aggregate works over the past two decades reinforces the impression that the fundamental impact of Stan's work is simply breathtaking.

Although research and disagreement continue on certain details of the cell death pathway, there are some principles that nearly all would agree on. We all agree that PCD is a genetically controlled pathway and that tumorigenesis involves its inhibition. The intrinsic pathway (and in some cases the extrinsic) is regulated by the BCL-2 family of proteins. The BCL-2 family of proteins may be divided into three groups the antiapoptotics, the multidomain proapoptotics, and the $\mathrm{BH} 3-$ only proteins. $\mathrm{BAX}$ and $\mathrm{BAK}$ are required for execution of a death signal in the intrinsic pathway, and operate downstream of the $\mathrm{BH} 3$-only proteins. The mitochondrion is an essential organelle in the control of PCD. Calcium homeostasis at the ER and death signaling originating at the ER are controlled by BCL-2 family members. Post-translational modification of BCL-2 family members can dramatically affect their function in the PCD pathway. It is remarkable that we are indebted to Stan Korsmeyer for the critical seminal observations leading to our understanding of every principle listed above. Below we outline what we consider to be some of the pillars that Stan erected to support the field's research into PCD.

\section{Genetic Control of Cell Death and Cancer}

In the benighted early 1980 s, it had not yet been settled that cell death was a program under genetic control. The story that the mammalian apoptotic pathway is governed genetically by the BCL-2 family began when BCL-2 was cloned at the breakpoint of the infamous $t(14 ; 18)$ translocation found in follicular lymphoma, reported by Stan's group and two other groups in $1985 .{ }^{1-3}$ It was later found that this novel protein blocked cell death following multiple toxic stimuli. ${ }^{4,5}$ This finding demonstrated that PCD was under genetic control, that BCL-2 was a critical control element and that inhibition of apoptosis played a crucial role in transformation. This result dovetailed nicely with work that demonstrated that developmental cell death in Caenorhabditis elegans was controlled by a genetically encoded program ${ }^{6}$ that shared function and sequence with BCL-2 proteins. ${ }^{7,8}$

\section{BCL-2 is a Member of a Family}

After BCL-2 was cloned and initially characterized, subsequent work by other laboratories identified other key mammalian relatives, including BCL-X ${ }_{\mathrm{L}}{ }^{9} \mathrm{MCL}-1,{ }^{10} \mathrm{BFL}-1,{ }^{11}$ and $\mathrm{BCL}-\mathrm{w} .{ }^{12}$ Important viral homologues were also identified, including E1B $19 \mathrm{~K}$ protein in adenovirus, ${ }^{13}$ BHRF-1 in Epstein-Barr virus,${ }^{14}$ and a BCL-2 homolog in Kaposi sarcoma herpes virus. ${ }^{15}$

A major paradigm shift occurred with the identification of the first proapoptotic interacting relative of BCL-2, BAX, in Stan's laboratory. ${ }^{16} \mathrm{BAX}$ was first described simultaneously with the description of a proapoptotic splice variant of BCL-X, BCL- $\mathrm{X}_{\mathrm{S}}$, by Craig Thompson's group. ${ }^{9}$ BAX was the first member identified of what would subsequently be known as the multidomain proapoptotic BCL-2 family proteins. It was also the first indication that adjudication of death decisions was performed via the interaction of pro- and antiapoptotic BCL-2 family members. The rheostat model, based on the knowledge of the existence only of BCL-2 and BAX, proposed that the decision to commit to programmed cell death was based on the relative abundance of these two key arbiters. BAK and BOK were subsequently cloned to fill out the trio of multidomain proapoptotic proteins identified to date.

The tidy rheostat model in its simplest two-molecule form was short-lived. Two years after discovering BAX, Stan foisted a critical modification of the model onto the field. $\mathrm{BAD}$, a protein that shared only the $\mathrm{BH} 3$ region of homology with previously identified family members, was found to act as a prodeath molecule. ${ }^{17}$ It apparently acted to displace BAX from $B C L-X_{L}$, so that the control of cell death was more involved than mere competition between antiapoptotic molecules and BAX. This report also founded the third class of the BCL-2 family, the so-called BH3-only proteins. Roughly 10 other $\mathrm{BH} 3-$ only proteins subsequently have been discovered, and the function of $\mathrm{BH} 3$-only proteins in controlling PCD is an area of very active research. The absence of a long homology region holds open the possibility of several more yet to be discovered, despite our complete knowledge of the human genomic sequence. 


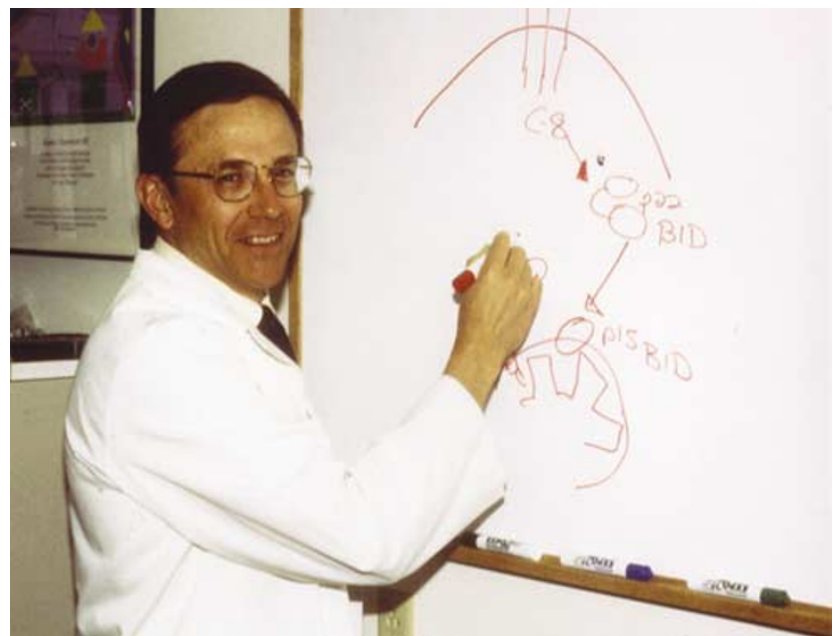

Figure 1 Stan Korsmeyer joyfully explaining an aspect of programmed cell death

\section{Mitochondria are Key Players in Cell Death}

We take it as given now that mitochondria play an indispensable role in adjudicating and executing death decisions. Until 1990, the mitochondrion was thought of as merely the cellular energy plant. The first hint that mitochondria might have an important role to play in PCD came with the observation from Stan's laboratory that BCL-2 is localized largely to the mitochondrion. ${ }^{18}$ It soon became clear that many other BCL-2 family members were similarly localized. Much superb work by several other laboratories subsequently demonstrated that this puzzling localization was not serendipitous. Release of mitochondrial cytochrome $c$ and other factors was critical to the eventual activation of caspases controlled by the BCL-2 family. The contribution of Stan's laboratory extends to our current understanding of the mechanisms by which proapoptotic members of the family recruit the organelle in the death program. He showed a role for mitochondrial shape changes in apoptosis; ${ }^{19}$ contributed to connecting glycolysis to cell death; ${ }^{20}$ showed that apoptotic mitochondria are reversibly dysfunctional due to the loss of cytochrome $c$. $^{21}$ All these discoveries clarified that BCL-2 family members are multifunctional proteins that engage multiple pathways to kill.

\section{BAX or BAK are Required to Execute a Death Decision Downstream of BH3-only Proteins}

It is now clear that $\mathrm{BAX}$ or $\mathrm{BAK}$ are required for intrinsic $\mathrm{PCD}$ in mammalian cells. This was not always so clear, especially given the absence of a BAX-like molecule in $C$. elegans. The clearest demonstration of their requirement resulted from a fruitful collaboration of Stan's with Craig Thompson's lab, when it was shown that compound knockouts of BAX and BAK were resistant to multiple apoptotic stimuli. ${ }^{22,23}$ Furthermore, the prodeath function of $\mathrm{BH} 3-$ only proteins relied on the presence of BAX or BAK. Stan subsequently showed that the mechanism underlying this requirement was the oligomerization of these proteins, which was required for the permeabilization of the mitochondrial outer membrane that was required for cytochrome $c$ release. ${ }^{24}$ While BAX has the ability to form pores that permit the egress of cytochrome $c$, the content of the in vivo cytochrome $c$ pore is still not completely resolved. ${ }^{25}$ There are indications, however, that BAX and BAK may indeed make up an important part of it. ${ }^{26}$

How are BAX and BAK triggered to release cytochrome $c$ ? Here, we enter an area of less complete consensus. Work from Stan's laboratory showed that interaction with the BH3only family member BID resulted in conformational changes and oligomerization of BAX and BAK, an interaction that can be prevented by antiapoptotic family members. ${ }^{24,27}$ This has provoked a model wherein a subset of BH3-only family members can directly activate BAX and BAK, whereas the remaining molecules act as antagonists of antiapoptotic proteins. $^{28}$ Moreover, Stan showed that BAX and BAK regulate not only the 'classical' pathway of death, but also $\mathrm{Ca}^{2+}$-dependent apoptosis. $\mathrm{Ca}^{2+}$ was known for a long time to participate in the amplification of death signals ${ }^{29}$ and levels of $\mathrm{BCL}-2$ also regulate $\mathrm{Ca}^{2+}$ levels in the endoplasmic reticulum. ${ }^{30-32} \mathrm{BAX}$ and $\mathrm{BAK}$ do the same to ultimately control apoptosis by stimuli that require $\mathrm{Ca}^{2+} \cdot{ }^{33}$

\section{Post-Translational Modification Controls Activity of Many BH3-only Proteins}

Once it became clear that $\mathrm{BH} 3$-only proteins played a vital role in modulating PCD, it became important to understand how the activity of these molecules was controlled. In the case of BID, work by Xiaodang Wang's lab as well as Stan's showed how caspase-8-mediated cleavage of BID induced cytochrome $c$ release in response to death receptor ligation. ${ }^{34,35}$ This key finding linked the extrinsic to the intrinsic cell death pathway. Work in the Korsmeyer lab subsequently showed that post-cleavage $N$-myristoylation of the p15 fragment further activated BID, assisting its membrane targeting. ${ }^{36}$

Phosphorylation of BAD was shown to play an important role in its activation. In response to survival factor signaling, BAD is phosphorylated, promoting its cytoplasmic sequestration by $14-3-3 .^{37}$ However, when survival signaling fails, $B A D$ is dephosphorylated and freed to act as an antagonist to antiapoptotic proteins like BCL-2. The observation that phosphorylation could modulate cell death made the important connection between the myriad kinase-mediated signaling pathways and the central death decision-making machinery.

As one reads the many excellent contributions to this issue from investigators all over the world, it is gratifying to note how many of them pay direct or indirect tribute to Stan by following trails that he blazed. Although Stan is gone, his work continues as long as the molecular study of PCD continues. The understanding of PCD has increased phenomenally in just over two decades. If we see further because we stand on the shoulders of giants, then Stan was certainly one of our tallest giants. 
1. Bakhshi A et al. (1985) Cell 41: 899-906.

2. Cleary ML and Sklar J (1985) Proc. Natl. Acad. Sci. USA 82: 7439-7443.

3. Tsujimoto $Y$ et al. (1985) Science 229: 1390-1393.

4. Vaux DL et al. (1988) Nature 335: 440-442.

5. McDonnell TJ et al. (1989) Cell 57: 79-88.

6. Ellis HM and Horvitz HR (1986) Cell 44: 817-829.

7. Hengartner MO and Horvitz HR (1994) Cell 76: 665-676.

8. Vaux DL et al. (1992) Science 258: 1955-1957.

9. Boise LH et al. (1993) Cell 74: 597-608.

10. Kozopas KM et al. (1993) Proc. Natl. Acad. Sci. USA 90: 3516-3520.

11. Choi SS et al. (1995) Oncogene 11: 1693-1698.

12. Gibson L et al. (1996) Oncogene 13: 665-675.

13. Rao L et al. (1992) Proc. Natl. Acad. Sci. USA 89: 7742-7746.

14. Pearson GR et al. (1987) Virology 160: 151-161.

15. Cheng EH et al. (1997) Proc. Natl. Acad. Sci. USA 94: 690-694.

16. Oltvai ZN et al. (1993) Cell 74: 609-619.

17. Yang E et al. (1995) Cell 80: 285-291.

18. Hockenbery D et al. (1990) Nature 348: 334-336.

19. Scorrano L et al. (2002) Dev. Cell 2: 55-67.
20. Danial NN et al. (2003) Nature 424: 952-956.

21. Mootha VK et al. (2001) EMBO J. 20: 661-671.

22. Wei MC et al. (2001) Science 292: 727-730.

23. Lindsten T et al. (2000) Mol. Cell 6: 1389-1399.

24. Wei MC et al. (2000) Genes Dev. 14: 2060-2071.

25. Saito M et al. (2000) Nat. Cell Biol. 2: 553-555.

26. Dejean LM et al. (2005) Mol. Biol. Cell. 16: 2424-2432.

27. Cheng EH et al. (2001) Mol. Cell 8: 705-711.

28. Letai A et al. (2002) Cancer Cell 2: 183-192.

29. Baffy G et al. (1993) J. Biol. Chem. 268: 6511-6519.

30. Foyouzi-Youssefi R et al. (2000) Proc. Natl. Acad. Sci. USA 97: 5723-5728.

31. Lam M et al. (1994) Proc. Natl. Acad. Sci. USA 91: 6569-6573.

32. Pinton $\mathrm{P}$ et al. (2001) EMBO J. 20: 2690-2701.

33. Scorrano $L$ et al. (2003) Science 300: 135-139.

34. Gross A et al. (1999) J. Biol. Chem. 274: 1156-1163.

35. Luo $X$ et al. (1998) Cell 94: 481-490.

36. Zha J et al. (2000) Science 290: 1761-1765.

37. Zha J et al. (1996) Cell 87: 619-628. 